\title{
From Localised to Ballistic Excitons in GaAs Quantum Wells
}

\author{
F. Pulizzi, P.C.M. Christianen, J.C. MaAn \\ High Field Magnet Laboratory, Research Institute for Materials \\ University of Nijmegen, Toernooiveld 1, 6525ED Nijmegen, The Netherlands \\ S. Eshlaghi, D. Reuter and A.D. Wieck \\ Angewandte Festköperphysik, Ruhr-Universität Bochum \\ 44780 Bochum, Germany \\ The lateral motion of excitons in GaAs quantum wells is studied by \\ means of spatially resolved photoluminescence. We show that at low tem- \\ peratures $(4.2 \mathrm{~K})$ the exciton motion evolves from localised excitons (zero \\ mobility) in thin quantum wells to extremely high mobilities in wide wells. \\ We find that for the widest quantum well investigated the observed motion \\ cannot be explained by simple exciton diffusion and must be explained by \\ the propagation of ballistic exciton polaritons.
}

PACS numbers: 71.35.-y, 71.36.+c

\section{Introduction}

The low temperature optical properties of semiconductor quantum wells (QWs) are dominated by bound electron-hole pairs (excitons). In contrast to electrons and holes, electric fields do not affect the motion of neutral excitons, which makes the study of their transport properties a difficult task. Several techniques have been used in the past to study the exciton motion, like the Raman spectroscopy [1], space and time resolved photoluminescence (PL) spectroscopy [2, 3], and transient grating methods [4]. According to these studies, the motion of excitons at low temperatures $(4 \mathrm{~K})$ is limited by scattering due to roughness of the QW interfaces, leading to exciton mobilities lower than $1 \mathrm{~m}^{2} /(\mathrm{V} \mathrm{s})$. This number suggests that during its lifetime an exciton could never travel a distance which can be resolved by far fields optics $(\approx 1 \mu \mathrm{m})[5]$.

In this paper we demonstrate, however, that for high quality QWs and under the proper experimental conditions, it is possible to record the low temperature exciton mobility, utilising a spatially resolved PL technique which measures 
the exciton motion after point-like excitation. We have found that the exciton mobility increases with increasing well width due to reduced interface roughness scattering. For the widest QW we find that photoexcited excitons can travel over distances larger than $5 \mu \mathrm{m}$ before recombination, corresponding to mobilities in excess of $10^{2} \mathrm{~m}^{2} /(\mathrm{V} \mathrm{s})$. In this particular case the exciton motion should be described in terms of the ballistic propagation of exciton polaritons, that is coupled exciton-photon modes.

\section{Experimental details}

The sample under study contains several GaAs QWs of different widths $L=5.1,8.3,12.2,19.8 \mathrm{~nm}$, grown by molecular beam epitaxy on a GaAs substrate and separated by thin AlAs/GaAs barriers. The beam of a tuneable continuous wave Ti:sapphire laser was focused on the sample surface by a $40 \times$ microscope objective. The resulting laser spot has a $1.2 \mu \mathrm{m}$ diameter, which is very close to the Rayleigh limit, which corresponds to a resolution of $0.7 \mu \mathrm{m}$. The PL emitted by the recombining excitons was collected by the same objective, focused on the entrance slit of a monochromator and imaged on a CCD camera. The resulting PL image is spectrally (spatially) resolved along the $x$-axis and spatially along the $y$-axis [6].

The measurements were performed using resonant excitation of light-hole $(\mathrm{LH})$ excitons and very low laser powers $\left(<10^{2} \mathrm{~W} / \mathrm{cm}^{2}\right)$ corresponding to an exciton concentration of about $10^{7} \mathrm{~cm}^{-2}$. As we have pointed out earlier [6], only under these special conditions, which have not been fulfilled in earlier experiments [2-4], it is possible to study the motion of non-interacting cold excitons in the absence of free electron and holes.

\section{Experimental results}

The PL images of the four QWs measured at $4.2 \mathrm{~K}$ are displayed in Fig. 1. For comparison we have also depicted the real space reflection of the laser spot (left panel). By taking the cross-sections of the pictures, we can extract both the spectral shape of the PL emitted from a given point of the sample (vertical cross-sections, aside of each panel) and the spatial profile of the PL emitted at a specific photon energy (horizontal cross-sections, below each panel). We first focus our attention on the spectral features of the PL. The very narrow line width $(0.25 \mathrm{meV})$ of the $19.8 \mathrm{~nm} \mathrm{QW}$ indicates the very high quality of this sample. With decreasing QW width we observe a broadening of the spectral line, as expected due to a stronger influence of well width fluctuations and due to interface roughness. The spectrum of the $5.1 \mathrm{~nm}$ QW consists of a $5.5 \mathrm{meV}$ broad background, and many narrow lines $(\approx 0.2 \mathrm{meV}$ width, which corresponds to the resolution of the monochromator) representing the localisation of excitons in naturally formed quantum dots at the QW interface [7]. 


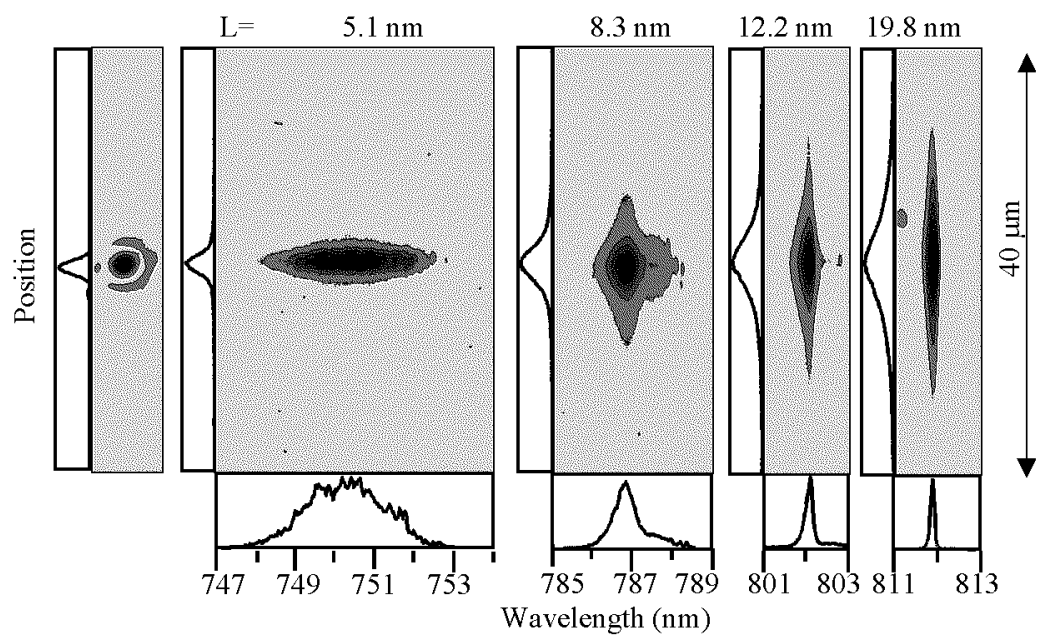

Fig. 1. PL images for the four QWs studied and the real space reflection of the laser spot (left panel). The graphs at the bottom of each picture represent the PL spectra emitted in the centre of the picture. The graphs on the left side of each picture are the spatial profiles of the PL in correspondence to the energy peak position.

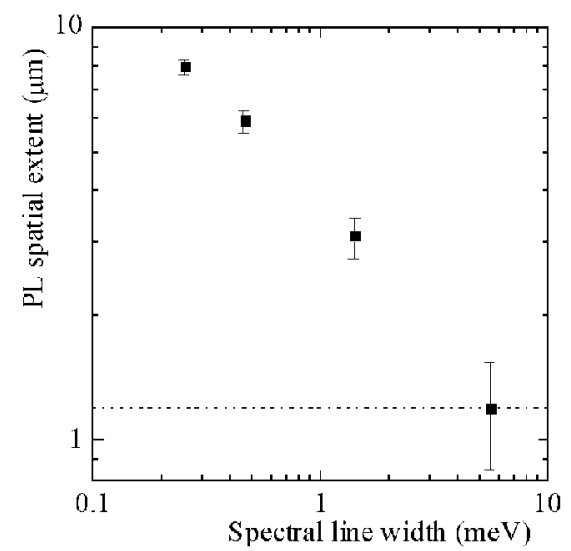

Fig. 2. Spatial extent of the PL profile as a function of the spectral line width of the different QWs. The dotted line represents the extent of the laser profile.

The localised nature of excitons in the $5.1 \mathrm{~nm}$ QW is confirmed by the coincidence of the PL spatial profile and the laser profile. In the other QWs the extent of the spatial profile is considerably larger than the laser, which demonstrates that the excitons are free and mobile. Moreover, we observe a one-to-one relationship between the spectral line width (and therefore QW thickness) and the spatial extent (Fig. 2). With increasing well width the spatial extent broadens up to $8 \mu \mathrm{m}$ (FWHM) for the $19.8 \mathrm{~nm} \mathrm{QW}$. 


\section{Discussion}

In order to analyse quantitatively the lateral motion of single excitons, we consider the simple steady state diffusion equation

$$
D \nabla^{2} n(x)+I(x)-n(x) / \tau=0,
$$

where $D$ is the diffusion constant, $n(x)$ is the exciton concentration, which we assume to be proportional to the local PL intensity, $\tau$ is the exciton lifetime and $I(x)$ is the exciton injection per time unit, which is taken to be proportional to the measured laser profile. We solved the equation numerically using $D$ as a free parameter and a fixed recombination time $\tau=600$ ps. ${ }^{*}$ In Fig. 3 the measured spatial profiles (symbols) for each QW together with the best fits (dashed lines) obtained with the diffusion model are shown. No fit is depicted for the $5.1 \mathrm{~nm}$ QW, as the profile coincides with the laser profile (dotted line). In the case of the $8.3 \mathrm{~nm}$ and $12.2 \mathrm{~nm}$ QWs, the profile can be well described by the diffusion model, with, respectively, $D=0.01 \mathrm{~m}^{2} / \mathrm{s}$ and $D=0.05 \mathrm{~m}^{2} / \mathrm{s} . D=0.05 \mathrm{~m}^{2} / \mathrm{s}$ corresponds to a very high mobility $\left(\mu \approx 10^{2} \mathrm{~m}^{2} /(\mathrm{V} \mathrm{s})\right)$ which is much higher than any previous experimental observation, and is very close to the upper limit of theoretical predictions at $4.2 \mathrm{~K}[3,8]$.

In the case of the $19.8 \mathrm{~nm} \mathrm{QW}$, the simple diffusion model cannot describe the experimental curve. The reason for this discrepancy can be understood quite easily. The diffusion equation can be used on a length scale which is long compared to the mean free path. If excitons hardly collide during their lifetime, the diffusion model should obviously break down. This condition is met when the average diffusion velocity $v_{\text {diff }}$ is comparable with the thermal velocity $v_{\mathrm{T}}$ [9]. The actual value of $v_{\text {diff }}$ can be estimated by $v_{\text {diff }}=R / \tau$, where $R$ is the spatial extent of the measured PL image. Using $R \approx 4 \mu \mathrm{m}$ we obtain $v_{\text {diff }}=8 \times 10^{3} \mathrm{~m} / \mathrm{s}$ which is comparable to $v_{\mathrm{T}} \approx 12 \times 10^{3} \mathrm{~m} / \mathrm{s}$ at $4 \mathrm{~K}$, using an exciton mass of $0.45 m_{0}$. The exciton motion in the $19.8 \mathrm{~nm} \mathrm{QW}$ is therefore clearly non-diffusive and in order to properly describe the experimental PL profile we have simulated the motion by means of a Monte Carlo method. In this calculation we assume that excitons start moving from the excitation region with a thermally distributed velocity, and use the exciton lifetime $\tau$ and scattering time $\tau_{\text {scatt }}$ as free parameters. The solid line in Fig. 3 is the result of the simulation using $\tau=600 \mathrm{ps}$ and $\tau_{\text {scatt }}=360 \mathrm{ps}$. It should be noted that the choice of these two parameters is unique, because $\tau$ determines the width of the curve, whereas $\tau / \tau_{\text {scatt }}$ determines the shape of the curve ${ }^{\dagger}$. From the perfect agreement between the experimental trace and the simulation we can

*The spatial extent of the solution of the diffusive equation $n(x)$ depends only on the product $D \tau$. The purpose of this paper is to find the right order of magnitude of the diffusivity; therefore we have chosen a reasonable lifetime for GaAs QW at $4.2 \mathrm{~K}$ (see for example J. Feldmann, G. Peter, E.O. Göbel, P. Dawson, K. Moore, C. Foxon, R.J. Elliot, Phys. Rev. Lett. 59, 2337 (1987)).

${ }^{\dagger}$ In case $\tau / \tau_{\text {scatt }}>3$ the diffusive profile is recovered. For the $12.2 \mathrm{~nm}$ and $8.3 \mathrm{~nm}$ we find respectively $\tau / \tau_{\text {scatt }}=4$ and $\tau / \tau_{\text {scat }}=20$. 


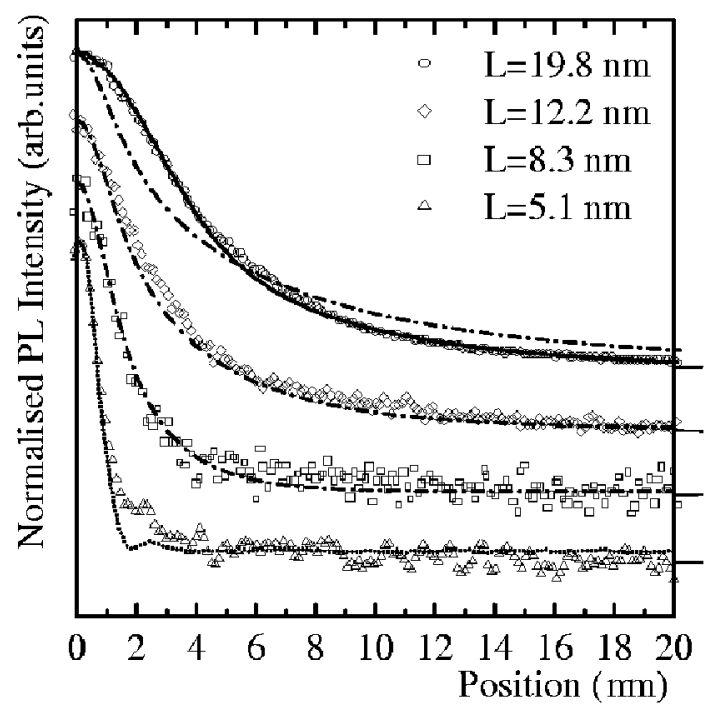

Fig. 3. Spatial profiles of the measured QWs (circles). For each profile the origin of the $y$-axis is represented by the short tick on the right axis. The dotted line is the laser profile. The dashed line is the diffusion fit and the solid line is the result of the Monte Carlo simulation explained in the text.

conclude that the observed motion has a ballistic character. However, the picture of ballistic motion must be an oversimplification, as pointed out below. Firstly, the mobility which corresponds to $\tau_{\text {scatt }}=360 \mathrm{ps}$ is $10^{3} \mathrm{~m} /(\mathrm{V} \mathrm{s})$, which is ten times bigger than the upper limit calculated theoretically [8]. Secondly, in our experiment we excite excitons resonantly, and therefore with a very small in-plane velocity. In order to thermalise and to acquire a velocity comparable to $v_{\mathrm{T}}$, as is used in the simulation, the excitons should scatter very rapidly with acoustic phonons, whereas experimentally the characteristic time is measured to be $100 \mathrm{ps}$ [10]. Thirdly, there is no reason to assume that the excitons reach the thermal distribution and then move ballistically without any scattering.

A possible explanation of the exceptionally large experimental PL extents could be that not bare excitons diffuse, but rather ballistic exciton polaritons. For polaritons the exciton and photon modes are coupled which may lead to very high group velocities, as is evidenced by recent time-of-flight experiments on microcavities [11]. The expected lifetime for polaritons in GaAs QWs is of the order of 40 ps [12], leading to an a verage velocity of $v_{\text {pol }}=R / \tau_{\text {pol }} \approx 10^{5} \mathrm{~m} / \mathrm{s}$. This value is one order of magnitude lower than the polariton group velocities measured in microcavities in the experiment of Ref. [10], but at the same time it is two orders of magnitude higher than the bare exciton velocity for resonant excitation. We emphasise that more theoretical work is needed to explain how the polariton coupling can occur so effectively in simple QWs. 


\section{Summary}

We have studied the low temperature exciton motion in GaAs QWs by means of spatially resolved photoluminescence spectroscopy, as a function of the QW width.

We can summarise our experimental findings as follows: in the $19.8 \mathrm{~nm} \mathrm{QW}$ exciton polaritons are excited, which, because of low scattering rates, propagate ballistically, leading to an exceptionally broad PL profile. With decreasing well width the interface scattering increases, and the exciton motion turns to be diffusive. Possibly also for the $12.2 \mathrm{~nm}$ QW a large contribution to the extent of the spatial profile should also be seen as exciton polaritons, which would explain the high value of the mobility found. With further decreasing well width $(8.3 \mathrm{~nm})$ the polaritonic propagation is suppressed and the high scattering rates allows the excitons to thermalise and move in a truly diffusive fashion. Eventually ( $L=5.1 \mathrm{~nm}$ ) the excitons are localised in natural quantum dots at the QW interface.

\section{References}

[1] K.T. Tsen, O.F. Sankey, G. Halama, Shu-Chen Y. Tsen, H. Morkos, Phys. Rev. B 39, 6276 (1989).

[2] W. Heller, A. Filoramo, Ph. Roussignol, U. Bockelmann, Solid State Electron. 40, 725 (1996).

[3] H. Hillmer, A. Forchel, S. Hansmann, M. Morohashi, E. Lopez, H.P. Meier, K. Ploog, Phys. Rev. B 39, 10901 (1989).

[4] J. Hegarty, L. Goldner, M.D. Sturge, Phys. Rev. B 30, 7346 (1984).

[5] B. Hanewinkel, A. Knorr, P. Thomas, S.W. Koch, Phys. Rev. B 60, 8975 (1999).

[6] F. Pulizzi, W.H.A. Thijssen, P.C.M. Christianen, J.C. Maan, Physica B 298, 441 (2001).

[7] A. Zrenner, L.V. Butov, M. Hagn, G. Abstreiter, G. Böhm, G. Weimann, Phys. Rev. Lett. 72, 3382 (1994); D. Gammon, E.S. Snow, B.V. Shanabrook, D.S. Katzer, D. Park, Phys. Rev. Lett. 76, 3005 (1996).

[8] P.K. Basu, Partha Ray, Phys. Rev. B 44, 1844 (1991).

[9] K. Hess, Advanced Theory of Semiconductor Devices, Ed. Nick Holonyak Jr., Prentice and Hall, Englewood Cliffs, New Jersey 1988, p. 132.

[10] M. Vollmer, H. Giessen, W. Stolz, W.W. Rühle, L. Ghislain, V. Elings, Appl. Phys. Lett. 74, 1791 (1999).

[11] T. Freixanet, B. Sermage, A.Tiberj, R. Planel, Phys. Rev. B 61, 7233 (2000).

[12] S. Jorda, U. Rössler, D. Broido, Phys. Rev. B 48, 1669 (1993). 